Weitere Infos auf springermedizin.de

Diabetes

Neue Leitlinien, neue Zielwerte, neue Antidiabetika? Mit den ständig aktualisierten SpringerMedizin-Dossiers bleiben Sie auf dem Laufenden.

-www.springermedizin.de/ api-diabetes

Rechtsmedizin

Selbst guillotiniert, 62 HeparinSpritzen injiziert oder vom

Dönerspieß erstochen - gehen Sie auf Spurensuche in kuriosen Fällen aus der Rechtsmedizin und lernen Sie ungewöhnliche Todesumstände und postume Diagnosen kennen

- www.springermedizin.de/ api-rechtsmedizin

Spektakuläre (Un-)Fälle

Es gibt Fälle, da muss auch der erfahrene Arzt erst mal schlucken: So der Patient, der sich mit Rückenschmerzen vorstellt; erst beim Entkleiden offenbart sich die Wurzel des Übels - ein rucksackgroßer Tumor. Manchmal sind es auch die „fiesen" kleinen Verletzungen, bei den man kaum hinsehen mag, z. B. der Finger der Hausfrau, der im Mixer feststeckt. Die Devise heißt in jedem Fall: Nerven behalten. Wie die Kollegen vorgegangen sind, zeigen die folgenden Beiträge. > www.springermedizin.de/ api-unfaelle

Notfallchecklisten für die Praxis Als Hausarzt sind Sie die erste Anlaufstelle für jeden erdenklichen Notfall. Da ist es manchmal schwer, immer gleich das richtige Konzept parat zu haben. Die praktischen Notfallchecklisten geben Ihrem Handeln einen roten Faden. - www.springermedizin.de/ api-notfallcheckliste

\title{
Nierentransplantation weltweit: buntes Spektrum mit Problemen
}

Nicht nur die Zahl, auch die Art der Nierentransplantationen schwankt in einzelnen Ländern ganz erheblich. Weil diese teils eigenartigen regionalen Unterschiede problematische und unethische Entwicklungen begünstigen, verlangt die WHO weltweit ein einheitliches Regelwerk.

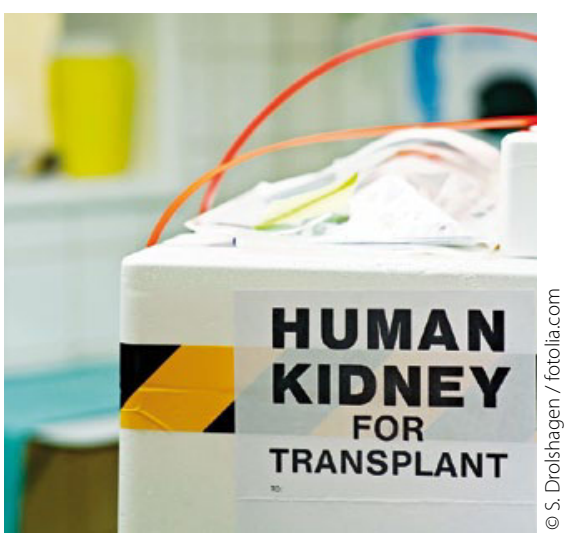

Die WHO will Organspenden weltweit einheitlich regeln.

— Nach WHO werden in 98 Ländern Organtransplantationen durchgeführt. Bei den Nierentransplantationen wurden 2009 in 22 Ländern nur Lebendnieren verpflanzt: in Jordanien 30 pro 1 Mill. Einwohner, gefolgt von Island, Libanon, Mauritius, Sri Lanka, Syrien,
Ägypten, Libyen, Oman, Pakistan und El Salvador (5).

Leichennieren wurden am häufigsten in Portugal (50), Spanien, Österreich, Frankreich, Belgien, Norwegen, Estland, Uruguay, Kroatien, Irland und USA (32) übertragen. Die Rangfolge der Länder mit den meisten Transplantationen verschiebt sich allerdings, wenn die in den einzelnen Ländern sehr unterschiedliche Zahl an Lebendnierentransplantationen mitgezählt wird: Norwegen (60), Portugal, USA, Holland, Schweden, England und Dänemark (42). Deutschland steht in dieser Rangliste an 21. Stelle mit 26 Leichen- und 7 Lebendnierentransplantationen pro 1 Million Einwohner.

\section{- F. L. Delmoniko et al.}

A call for government accountability to achieve national self-sufficiency in organ donation and transplantation. Lancet 378 (2011) 1414-1418

\section{Kommentar}

Die Zahl der Organtransplantationen schwankt erheblich, und in etwa der Hälfte der Länder dieser Erde wird überhaupt nicht transplantiert. Hinzu kommen die Unterschiede in der Art der Transplantationen. Nach Ansicht der WHO begünstigen diese Tatsachen Organhandel und Transplantationstourismus, ohne dass bestimmten Merkmalen der Statistik besondere Probleme zugeordnet werden. Die WHO verlangt die Entwicklung nationaler Programme und Vorschriften für Organspenden und deren Transplantation, damit alle Nationen den Transplantationsbedarf der eigenen Bevölkerung selbst abdecken können (Self-sufficiency) und damit ethische Probleme für Organspender und -empfänger vermieden werden. Das Regelwerk muss u.a. festschreiben, dass
Lebendspender genetisch, gesetzlich und emotional mit dem Empfänger verbunden sind, dass Minderjährige und Personen mit eingeschränkter Zustimmungsfähigkeit vor Organspenden geschützt werden, dass Organspenden freiwillig ohne Bezahlung und Belohnung erfolgen müssen, dass die Zuteilung von Organen nach unparteiischen, rechtmäßigen und ethischen, keinesfalls nach finanziellen oder anderen Gesichtspunkten erfolgen darf. Auch ein heikles Thema mit offensichtlichem, aber nicht explizit genanntem religiösen Bezug wird angesprochen: Eine Person, die Empfänger eines Leichenorgans sein darf, muss auch Spender sein können und umgekehrt.

H. Holzgreve 